\title{
Strafrechtlicher Schutz des E-Mail-Verkehrs post mortem?
}

Der Umgang mit dem digitalen Nachlass ist eine Frage von zunehmender juristischer Bedeutung. Hat der Erblasser keine Vorkehrungen getroffen, so stellt sich aus strafrechtlicher Sicht etwa die Frage, ob sich E-Mail-Provider bei der Herausgabe von Zugangsdaten zu den E-MailAccounts des Erblassers strafbar machen. Eine Analyse einschlägiger Strafbestimmungen zeigt, dass eine allfällige Strafbarkeit der Provider grundlegend von der Erbenstellung determiniert wird und eine Strafbarkeit bei der Herausgabe von Zugangsdaten an Erben ausser Betracht fällt.

I. Einleitung

II. Zugänglichmachen des E-Mail-Verkehrs post mortem: Strafbarkeit der Provider?

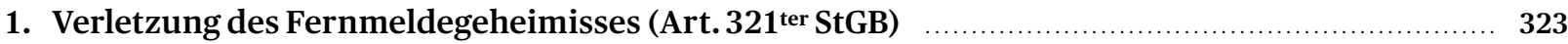

2. Unbefugtes Eindringen in ein Datenverarbeitungssystem (Art. 143 ${ }^{\text {bis }}$ StGB) $\ldots \ldots \ldots \ldots \ldots \ldots \ldots \ldots$

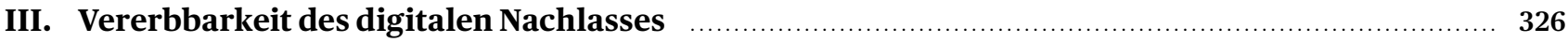

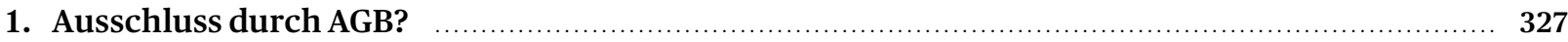

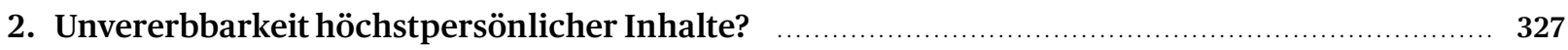

3. Fortbestand des Fernmeldegeheimnisses gegenüber Kommunikationspartnern? $\quad \ldots \ldots \ldots \ldots \ldots \ldots$

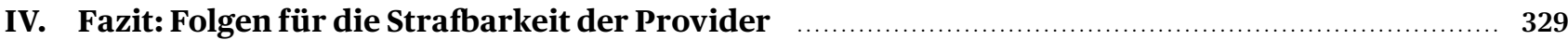

Zitiervorschlag:

ELENA BIAGGINI, Strafrechtlicher Schutz des

E-Mail-Verkehrs post mortem?, sui generis 2021, S. 321

Elena Biaggini, MLaw, LL.M., Assistentin und Doktorandin am Lehrstuhl für Strafrecht und Strafprozessrecht von Prof. Dr. iur. Sarah Summers an der Universität Zürich (elena.biaggini@rwi.uzh.ch). Die Autorin bedankt sich bei Alen Gorgievski, MLaw, LL.M., und Michael Weber, MLaw, für die kritische Durchsicht des Manuskripts und die wertvollen Anmerkungen.

URL: sui-generis.ch/196

DOI: https://doi.org/10.21257/sg.196

Dieses Werk ist lizenziert unter einer Creative Commons Namensnennung - Weitergabe unter gleichen Bedingungen 4.0 International Lizenz. 


\section{Einleitung}

1 Im heutigen digitalen Zeitalter verfügt fast jede Person über mindestens einen E-Mail-Account. Wohl die wenigsten InhaberInnen setzen sich indessen zu Lebzeiten mit einer Regelung dieses «digitalen Nachlasses» auseinander, sodass sich die Frage stellt, ob und in welchem Umfang für Hinterbliebene ein Zugangsrecht zu diesem besteht.

2 Auf Seiten der Erben können sowohl persönliche ${ }^{1}$ als auch vermögensrechtliche Interessen bestehen, auf die E-Mail-Accounts des Erblassers zugreifen zu können. Verschiedenste Vertragsverhältnisse werden heutzutage online abgeschlossen und Rechnungen oftmals per E-Mail empfangen. ${ }^{2}$ Daneben kommt der E-Mail-Adresse im technischen Verkehr häufig Legitimationscharakter zu, wie etwa beim E-Banking-Dienst PayPal. ${ }^{3}$ Auch Passwörter lassen sich häufig nur über eine E-Mail-Adresse zurücksetzen. ${ }^{4}$ Schliesslich kann auch die Löschung des Accounts den Zugriff erforderlich machen. ${ }^{5}$ Der Zugang zum E-Mail-Account kann somit für die Nachlassverwaltung von erheblicher Bedeutung sein. ${ }^{6}$

3 Indes scheint eine gewisse Rechtsunsicherheit zu bestehen, ob E-Mail-Provider («Provider») den Angehörigen Zugang zum E-Mail-Account des Erblassers gewähren dürfen oder sich hierdurch gar strafbar machen. Der Blick auf die Praxis offenbart, dass manche Provider derzeit unter Vorweis einer Erbbescheinigung oder Sterbeurkunde die Kündigung des Vertrags oder eine AccountÜbernahme zulassen, ${ }^{7}$ während der Herausgabe von Zugangsdaten vormals zuweilen Geheimhaltungspflichten wie namentlich das Fernmeldegeheimnis entgegengehalten wurden. ${ }^{8}$

1 So etwa eine Mutter, welche den Zugriff auf das Facebook-Konto ihrer verstorbenen Tochter in Deutschland gerichtlich erwirken musste, siehe CORDULA LÖTSCHER, Facebook-Status: verstorben, NZZ vom 9. März 2021, S. 10 (zit. LÖTSCHER, NZZ).

2 MARTINA BOSSHARDT / LORENA BUR, Nachlassplanung und Nachlassabwicklung, in: Hrubesch-Millauer/Wolf/Eggel(Hrsg.), Digitales Erbrecht - Perspektiven aus der Schweiz, Bern 2021, S.179.

3 NAHUEL GuidotTI, in: Hrubesch-Millauer/Wolf/Eggel(Hrsg.) Digi tales Erbrecht - Perspektiven aus der Schweiz, Bern 2021, S. 23 f.

4 Siehe HANS RAINER KÜNZLE, Digitaler Nachlass nach schweizerischem Recht, successio 2015, S. 43; BOSSHARDT/BUR (Fn. 2), S. 178.

5 Vgl. MELANIE STUDER / MATTHIAS SCHWEIZER / ELKE BRUCKERKLEY, Sterben und Erben in der digitalen Welt, Jusletter 17. Dezember 2012, N6 (zit. STUDER et al.).

6 So auch KÜNZLE (Fn. 4), S. 44.

7 So etwa bei UPC; bei einer Übertragung sind dabei die bislang auf dem Account gespeicherten Daten weiterhin einsehbar, siehe Formular zur Kündigung oder Kontoübertragung infolge Todesfall; ebenso die aktuelle Praxis von Sunrise und Swisscom, so auf telefonische Anfrage durch die Autorin; vgl. ferner auch Informationen von Swisscom im Todesfall.

8 Vgl. der Nachweis bei ELKE BRUCKER-KLEY / THOMAS KELLER/LUKAS KURTZ / KURT PÄRLI / MATTHIAS SCHWEIZER, Sterben und Erben in der digitalen Welt, Winterthur 2013, S. 56 (zit. BRUCKER-KLEY et al.); KÜNZLE (Fn. 4), S. 44 m.H.; ebenso STUDER et al. (Fn. 5), N 43; BOSSHARDT/BUR (Fn. 2), S. 183 .
Zunehmend befasst sich auch das Schrifttum insbeson- 4 dere mit dem Zugangsrecht der Erben auf den digitalen Nachlass ${ }^{9}$ aus erb- sowie datenschutzrechtlicher Sicht. ${ }^{10}$ Strafrechtliche Aspekte hierzu sind demgegenüber, soweit ersichtlich, bislang kaum vertieft behandelt worden.11

Vorliegend steht die Frage im Vordergrund, ob sich Pro- 5 vider durch die Herausgabe von Zugangsdaten zu E-MailAccounts verstorbener Personen an Erben oder Dritte strafbar machen (II.). Wie sich zeigen wird, ist hierbei eine Auseinandersetzung mit der Vererbbarkeit des digitalen Nachlasses unabdingbar (III.). Mit einer abschliessenden Darlegung der gewonnenen Erkenntnisse wird versucht, einen Beitrag zur Klärung der Rechtslage in Bezug auf die Strafbarkeit der Provider bei der Herausgabe von Zugangsdaten post mortem zu leisten (IV.). Vorliegend wird dabei von der Prämisse ausgegangen, dass der Erblasser keine Regelung hinsichtlich des postmortalen Umgangs mit seinen E-Mail-Accounts getroffen hat, ${ }^{12}$ dass es sich um einen inländischen Provider handelt, ${ }^{13}$ und nicht ein geschäftlicher, sondern ein privater E-MailAccount betroffen ist. ${ }^{14}$

\section{Zugänglichmachen des E-Mail-Verkehrs post mortem: Strafbarkeit der Provider?}

Die Frage, ob sich Provider bei der Herausgabe von Zu- 6 gangsdaten wie namentlich Passwörtern an Erben oder Dritte strafbar machen, gab soweit ersichtlich noch keinen

9 Für eine Begriffsdefinition siehe BRUCKER-KLEY et al. (Fn. 8), S.38; STEPHANIE HRUBESCH-MILlAUER, Der digitale Nachlass und die Frage nach einem Sondererbrecht, in: Hrubesch-Millauer/Wolf/Eggel (Hrsg.) Digitales Erbrecht - Perspektiven aus der Schweiz, Bern 2021, S. 124 ff.; KÜNZLE (Fn. 4), S. 39f.; CORDULA LÖTSCHER, Der digitale Nachlass, Zürich 2021, S. 3 ff (zit. LÖTSCHER, Der digitale Nachlass).

10 Siehe etwa BRUCKER-KLEY et al. (Fn. 8), passim; LÖTSCHER, Der digitale Nachlass (Fn.9), passim; CORDULA LÖTSCHER, Das erbrechtliche Schicksal von Accounts bei Facebook, Google, Apple \& Co., successio 2020, S. 312 m.w.H. (zit. LÖTSCHER, Das erbrechtliche Schicksal). Zur Frage nach einem Auskunftsrecht über Daten verstorbener Personen de lege lata und delegeferenda ferner LÖTSCHER, Der digitale Nachlass (Fn. 9), S.106ff.

11 In den Grundzügen diskutiert etwa bei BOSSHARDT/BUR (Fn. 2), S. 184 ff.; DANIEL HÜRLIMANN / SILVIO HÄNSENBERGER, Zugangsdaten zu Online-Accounts im Todesfall, in: Schweizerischer Notarenverband (Hrsg.), Aktuelle Themen zur Notariatspraxis 2020, Bern 2020, S. 76 f.; LÖTSCHER, Der digitale Nachlass (Fn. 9), S.169.

12 Überlässt der Erblasser den Erben die Zugriffsdaten, ist von einer faktischen Zugriffsgewährung auszugehen, siehe MARTIN EGGEL / NAHUEL GUIDOTTI / STEPHAN WOLF, Überlegungen zur rechtlichen Einordnung des «Digitalen» im Erbgang, in: Hrubesch-Millauer/ Wolf/Eggel (Hrsg.) Digitales Erbrecht - Perspektiven aus der Schweiz, Bern 2021, S. 94; vgl. hierzu auch PHILIPPE WEISSENBERGER, in: Niggli/Wiprächtiger (Hrsg.), Basler Kommentar, Strafrecht,

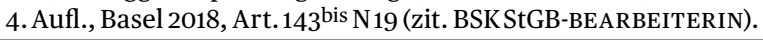

13 Vgl. zur Problematik von im Ausland ansässiger Provider HÜRLIMANN/HÄNSENBERGER (Fn.11), S. $77 \mathrm{f}$.

14 Zu arbeitsrechtlichen Implikationen vgl. etwa HÜRLIMANN/HÄNSENBERGER (Fn.11), S. 69 m.H. 
Anlass bundesgerichtlicher Klärung. Nachfolgend soll auf die möglicherweise einschlägigen Strafbestimmungen eingegangen werden.

\section{Verletzung des Fernmeldegeheimisses (Art.321 ${ }^{\text {ter }}$ StGB)}

7 Gemäss Art. $321^{\text {ter }}$ StGB15 machen sich Beamte, Angestellte oder Hilfspersonen von Post- oder Fernmeldedienstanbietern strafbar, welche Dritten i.S.v. Art. 43 FMG ${ }^{16}$ unter anderem Angaben über den Fernmeldeverkehr der Kundschaft machen, eine verschlossene Sendung öffnen oder ihrem Inhalt nachforschen oder einem Dritten Gelegenheit geben, eine solche Handlung zu begehen.

8 Strafbar ist zunächst, Dritten Angaben über den Fernmeldeverkehr zu machen. Der Terminus «Angaben» umfasst neben inhaltlichen Informationen zur Kommunikation auch andere Informationen, die sich auf das Kommunikationsverhalten beziehen (sog. Randdaten i.S.v. Art. 8 lit. b BÜPF 17), wie namentlich Kommunikationspartner sowie Art, Dauer und Häufigkeit der Kommunikation. ${ }^{18}$ Erfasst sind damit Angaben, welche unmittelbar die Kommunikation als solche betreffen. Die Preisgabe von Passwörtern lässt sich demgegenüber kaum hierunter fassen. ${ }^{19}$

9 Stattdessen ist vorliegend die dritte Tathandlungsvariante, einem Dritten Gelegenheit zu geben, die beschriebenen Handlungen vorzunehmen, von Interesse. Für die Tatbestandsmässigkeit ist unerheblich, auf welchem Weg dem Dritten die Möglichkeit der Informationserlangung verschafft wird. ${ }^{20}$ Die Preisgabe von Zugangsdaten wie Passwörtern lässt sich somit ebenfalls hierunter fassen. ${ }^{21}$ Erforderlich ist indes ein aktives Zutun seitens des Providers. Keine Strafbarkeit begründet das blosse Dulden der Zugriffnahme der Erben auf den Account, etwa wenn sie

15 Schweizerisches Strafgesetzbuch vom 21. Dezember 1937 (StGB; SR 311.0).

16 Fernmeldegesetz vom 30. April 1997 (FMG; SR 784.10).

17 Bundesgesetz betreffend die Überwachung des Post- und Fernmeldeverkehrs vom 18. März 2016 (BÜPF; SR 780.1).

18 BSK StGB-OBERHOLZER, Art. $321^{\text {ter N } 6 \text {; STEFAN TRECHSEL / VIKTOR }}$ LIEBER, in: Trechsel/Pieth (Hrsg.), Schweizerisches Strafgesetzbuch, Praxiskommentar, Zürich 2017, Art. 321 ${ }^{\text {ter }}$ N 2 (zit. PK StGB-BEARBEITERIN); MATTHIAS MICHLIG / EVA WYLER, in: Graf(Hrsg.), Annotierter Kommentar StGB, Art. 321 ${ }^{\text {ter }}$ N1 (zit. Komm. StGB-BEARBEITERIN).

19 Selbiges hat ferner in Bezug auf eine allfällige Strafbarkeit nach Art. 50 FMG zu gelten, da sich auch dieser nur auf inhaltiche Informationen bezieht.

20 Vgl. BSK StGB-OBERHOLZER, Art. $321^{\text {ter }}$ N6.

21 Zur Herausgabe von Passwörtern als tatbestandsmässige Handlung ohne genauere Qualifikation vgl. JÜRG SCHNEIDER, Internet Service Provider im Spannungsfeld zwischen Fernmeldegeheimnis und Mitwirkungspflichten bei der Überwachung des E-Mail-Verkehrs über das Internet, AJP 2005, S.188; in diesem Sinne wohl auch LÖTSCHER, Der digitale Nachlass (Fn. 9), S. 82; a.M. wohl PK StGB-TRECHSEL/ LIEBER, Art. 321 ${ }^{\text {ter }}$ N3, welche bei dieser Tatbestandsvariante von einer Unterlassung auszugehen scheinen. in den Besitz der Passwörter gelangt sind, weil der Erblasser sie auf einem Notizzettel hinterlassen hat. ${ }^{22}$

Unbestritten fällt neben den traditionellen Kommunika- 10 tionsformen grundsätzlich auch der E-Mail-Verkehr unter das verfassungsrechtlich in Art.13 Abs.1 BV 23 geschützte Fernmeldegeheimnis ${ }^{24}$ und ist folglich auch von Art. $321^{\text {ter }}$ StGB erfasst. ${ }^{25}$ Unerheblich ist, ob die Kommunikation privater oder geschäftlicher Natur ist oder ob die Übermittlung offen oder verschlüsselt erfolgt. ${ }^{26}$ Vom Fernmeldegeheimnis geschützt wird dabei die Vertraulichkeit der Kommunikation ${ }^{27}$ und somit nur der Kommunikationsvorgang selbst, ${ }^{28}$ nicht hingegen etwa das Zielmedium wie der E-Mail-Account. So greift der Schutz einzig für die Dauer des Übermittlungsvorgangs der Information ${ }^{29}$ und endet, sobald dieser abgeschlossen ist. ${ }^{30}$

\section{a) Abschluss des Kommunikationsvorgangs beim E-Mail-Verkehr}

Bei physischer Briefpost gilt der Kommunikationsvorgang 11 als abgeschlossen, sobald sich die Sendung im Briefkasten und damit im Machtbereich des Empfängers befindet, ungeachtet der Tatsache, ob dieser den Inhalt bereits zur Kenntnis genommen hat. ${ }^{31}$ Abgestellt wird folglich darauf, ob der Empfänger die Datenherrschaft erlangt und damit über das weitere Schicksal der übermittelten Information bestimmen kann. ${ }^{32}$

Für die Beurteilung der Dauer des Kommunikationsvor- 12 gangs beim E-Mail-Verkehr finden diese Überlegungen nach h.L. sinngemäss Anwendung. ${ }^{33}$ Der E-Mail-Verkehr

22 Siehe HÜRLIMANN/HÄNSENBERGER (Fn.11), S.79f.

23 Bundesverfassung der Schweizerischen Eidgenossenschaft vom 18. April 1999 (BV; SR 101).

24 GIOVANNI BIAGGINI, Bundesverfassung, Kommentar, 2. Aufl., Zürich 2017, Art. 13 N10; ANDREAS DONATSCH / ALBERT SCHMID, Der Zugriff aufE-Mails im Strafverfahren - Überwachung (BÜPF) oder Beschlagnahme?, in: Schwarzenegger/Arter/Jörg (Hrsg.), Internet-Recht und Strafrecht, 4. Tagungsband, Bern 2005, S. 152; PKStGB-TRECHSEL/ LIEBER, Art. 321 ${ }^{\text {ter }}$ N2; Botschaft vom 27. Februar 2013 zum Bundesgesetz betreffend die Überwachung des Post- und Fernmeldeverkehrs (BBl 20132683), S. 2704; vgl. auch BGE140 IV 181 E. 2.

25 Grundlegend BGE126I50 E. 6a; PK StGB-TRECHSEL/LIEBER, Art. 321 ${ }^{\text {ter }}$ N2; Komm. StGB-MICHLIG/WYLER, Art. $321^{\text {ter }}$ 1.

26 BIAGGINI (Fn. 24), Art.13 N10; OLIVER DIGGELMANN, in: Waldmann/ Belser/Epiney (Hrsg.), Basler Kommentar, Bundesverfassung, Basel 2015, Art. 13 N 29 (zit. BSK BV-BEARBEITERIN).

27 BSK BV-DIGGELMANN, Art.13 N29; siehe auch BGE126 I 50 E. 6 a.

28 DONATSCH/SCHMID (Fn. 24), S. 157; STUDER et al. (Fn. 5), N 43; siehe auch KÜNZLE (Fn. 4), S. 44; BGE140 IV181 E.2.4.

29 MARCJEAN-RICHARD-DIT-BRESSEL, Die Mailbox, Ziel oder Weg?, ZStrR 2007, S. 171; THOMAS HANSJAKOB, Überwachungsrecht der Schweiz, Zürich 2018, N301.

30 DONATSCH/SCHMID (Fn. 24), S. 157; JEAN-RICHARD-DIT-BRESSEL (Fn. 29), S.171.

31 Siehe DONATSCH/SCHMID (Fn. 24), S. 157; HANSJAKOB (Fn. 29), N302f

32 Vgl. BGE140 IV181 E. 2.5; HANSJAKOB (Fn. 28), N302f.

33 Vgl. DONATSCH/SCHMID (Fn. 24), S. 151 ff.; HANSJAKOB (Fn. 29), N313; JEAN-RICHARD-DIT-BRESSEL (Fn. 29), S.157ff. 
zeichnet sich allerdings durch folgende Besonderheiten aus: Dem Grundsatz nach wird eine empfangene E-Mail zunächst auf dem jeweiligen externen Server des Providers gespeichert. ${ }^{34}$ Verwendet der Empfänger auf seinem Computer oder Smartphone eine lokale E-Mail-Software wie Outlook oder Apple Mail, werden beim Abruf der E-Mails diese dort als Kopie lokal gespeichert. ${ }^{35}$ Jedenfalls ab dem Zeitpunkt, ab welchem sich die E-Mail (bzw. Kopie) auf der lokalen Software des Empfängers befindet, ist sie nicht mehr dem Fernmeldegeheimnis unterstellt. ${ }^{36}$

13 Wie sich der Umgang mit E-Mails gestaltet, die sich auf einem webbasierten E-Mail-Account bzw. dem externen Server des Providers befinden, auf welche der Provider ebenfalls noch eine theoretische Zugriffsmöglichkeit hat, wurde in Bezug auf die Zugriffsgewährung an Erben höchstrichterlich noch nicht entschieden. Sachgerecht erscheint es, in diesem Zusammenhang auf die ständige Rechtsprechung hinsichtlich der Abgrenzung zwischen strafprozessualer Beschlagnahme ${ }^{37}$ und geheimer Fernmeldeüberwachung ${ }^{38}$ zurückzugreifen. Hiernach bestimmt sich der Zeitpunkt, ab welchem der Schutzbereich des Fernmeldegeheimnisses endet, danach, ob der Empfänger sein E-Mail-Konto abgerufen, d.h. eine Verbindung mit dem Server des Providers hergestellt und vom Eingang der E-Mail (nicht jedoch zwingend auch vom Inhalt) Kenntnis erlangt hat. ${ }^{39}$ Der geschützte Datenübertragungsvorgang dauert damit so lange an, bis der Empfänger sein E-Mail-Konto geöffnet hat. ${ }^{40}$ Dies entspreche in Analogie zum Briefverkehr dem Öffnen des physischen Postfachs. ${ }^{41}$ Da der Empfänger ab diesem Zeitpunkt über die E-Mail verfügen kann, sei es unerheblich, ob er diese auch auf seinen internen Speicher heruntergeladen hat, sie löscht oder sie im Postfach auf dem externen Server des Providers belässt. ${ }^{42}$

14 Auf die vorliegende Konstellation angewandt folgt hieraus, dass die auf dem internen Speicher des Erblassers abrufbaren E-Mails ebenso wenig unter das Fernmeldegeheimnis fallen wie die bis zum letztmaligen Abrufen

34 HÜRLIMANN/HÄNSENBERGER (Fn.11), S. 69.

35 Siehe zum Ganzen HÜRLIMANN/HÄNSENBERGER (Fn.11), S. 69.

36 HANSJAKOB (Fn. 29), N313; sTUDER et al. (Fn. 5), N 43; siehe auch KÜNZLE (Fn. 4), S. 44.

37 Art. 263 der Schweizerischen Strafprozessordnung vom 5. Oktober 2007 (Strafprozessordnung, StPO; SR 312.0).

38 Art. 269 ff. StPO.

39 BGE140 IV181 E. 2.6; BGE143 IV 270 E. 4.6; BGE144IV 74 E. 2.4; für noch nicht gelesene E-Mails offen gelassen bei BRUCKER-KLEY et al. (Fn. 8), S. 56.

40 BGE140 IV181 E. 2.6; siehe auch BosshardT/BUR (Fn. 2), S. 184.

41 BGE140IV181 E. 2.6.

42 Siehe BGE140 IV 181 E. 2.6; differenziert bei нANSJAKoв (Fn. 29), N315f., nach welchem auch beim Herunterladen der E-Mail vom externen Server auf das lokale Gerät eine dem Fernmeldegeheimnis unterstehende Verbindung aufgebaut wird. des E-Mail-Kontos auf dem externen Server eingetroffenen E-Mails, unabhängig davon, ob diese auch vom Erblasser auf den internen Speicher heruntergeladen oder gelesen wurden. Für diejenigen E-Mails, welche bereits vor dem Ableben des Erblassers an diesen zugestellt wurden, stellt das Fernmeldegeheimnis auch aus strafrechtlicher Sicht kein Hinderungsgrund für die Zugriffsgewährung der Provider dar und eine Strafbarkeit nach Art. $321^{\text {ter }}$ StGB fällt a priori ausser Betracht. ${ }^{43}$

Für E-Mails, welche nach Ableben des Erblassers auf dem 15 E-Mail-Account eintreffen, dauert der durch das Fernmeldegeheimnis geschützte Übertragungsvorgang gemäss bundesgerichtlicher Rechtsprechung hingegen noch an. ${ }^{44}$

\section{b) Fernmeldegeheimnis über den Tod hinaus?}

Klärung bedarf ferner, ob das Fernmeldegeheimnis über 16 den Tod des Kunden hinaus Wirkung entfaltet, zumal etwa der Persönlichkeitsschutz mit dem Tod endet. ${ }^{45} \mathrm{Im}$ Schrifttum wird auf das Anwalts-, Arzt-, sowie das Bankgeheimnis verwiesen, welche ebenfalls nach dem Ableben der betroffenen Person fortwähren. ${ }^{46}$ Zur Vermeidung von Wertungswidersprüchen ist davon auszugehen, dass auch das Fernmeldegeheimnis nach dem Tod grundsätzlich fortbesteht. ${ }^{47}$ Dies muss umso mehr gelten, da das Fernmeldegeheimnis beide Kommunikationspartner und damit neben dem Erblasser auch die an der Kommunikation beteiligte Drittperson schützt. ${ }^{48}$ Davon zu trennen ist indessen, ob das Fernmeldegeheimnis ebenfalls gegenüber den Erben als Rechtsnachfolger des Erblassers besteht, worauf zurückzukommen ist. ${ }^{49}$

\section{c) E-Mail-Provider als Fernmeldeanbieter?}

Da Art. 321 $1^{\text {ter }}$ StGB als echtes Sonderdelikt ausgestaltet 17 ist, gilt es ferner zu untersuchen, ob Provider unter den Begriff der Fernmeldedienstanbieter fallen.

Das BÜPF unterscheidet grundlegend zwischen Fern- 18 meldedienstanbieterinnen i.S.v. Art. 3 lit.b FMG (Art. 2 lit. b BÜPF, «FDA») und sog. Anbieterinnen abgeleiteter Kommunikationsdienste, welche lediglich Dienste anbie-

43 Vgl. hierzu BOSSHARDT/BUR (Fn. 2), S.184; EGGEL/GUIDOTTI/WOLF (Fn.12), S.115; a.M. offenbar HÜRLIMANN/HÄNSENBERGER (Fn.11), S. 84; in diesem Sinne auch BRUCKER-KLEY et al. (Fn. 8), S. 56.

44 Vgl. hierzu BGE140IV181 E.2.6.

45 Siehe Art. 31 Abs. 1 des Schweizerischen Zivilgesetzbuches vom 10. Dezember 1907 (ZGB; SR 210); HÜRLIMANN/HÄNSENBERGER (Fn.11), S. 73 m.H.

46 HÜRLIMANN/HÄNSENBERGER(Fn.11), S.77; vgl. auch BRUCKER-KLEY et al. (Fn. 8), S. 54; RALPH GRAMIGNA / URS MAURER-LAMBROU, in Maurer-Lambrou/Blechta (Hrsg.), Basler Kommentar, Datenschutzgesetz/Öffentlichkeitsgesetz, 3. Aufl., Basel 2014, Art. 9 N19; zur Fortdauer des Anwaltsgeheimnisses über den Tod BSK StGB-OBERHOLZER, Art. 321 N18; BGE135 III 597.

47 Ebenso HÜRLIMANN/HÄNSENBERGER (Fn.11), S. 77.

48 So auch HÜRLIMANN/HÄNSENBERGER (Fn.11), S. 77 .

49 Siehe nachfolgend III.3. 
ten, die sich auf bestehende Fernmeldedienste stützen und eine Einweg- oder Mehrwegkommunikation ermöglichen (Art. 2 lit. c BÜPF, «AAKD»). Unter den Begriff der FDA fallen nur diejenigen Anbieter, welche eine fernmeldetechnische Übertragung, d.h. das Senden oder Empfangen von Informationen selbst anbieten. ${ }^{50}$ Dazu zählen namentlich die Swisscom, Sunrise oder UPC, welche den Teilnehmern Festnetz- und Mobiltelefonie sowie den Zugriff auf das Internet ermöglichen. ${ }^{51}$

Umstritten erschien bislang, ob Anbieterinnen von Diensten, welche über das Internet erbracht werden, selber aber keinen Internetzugang anbieten (sog. Over-the-TopDienste; «OTT-Dienste»), ebenfalls FDA oder AAKD darstellen. ${ }^{52} \mathrm{Zu}$ den OTT-Diensten zählen etwa reine E-MailProvider wie Gmail, Anbieterinnen von Speicherplatz für E-Mails oder Hoster von E-Mail-Diensten wie etwa GMX, welche selber kein Fernmeldenetzwerk betreiben, sondern auf bestehende Netzinfrastrukturen zurückgreifen. ${ }^{53}$ Die Unterscheidung ist unter dem BÜPF insbesondere daher von Bedeutung, weil sie den Umfang der Mitwirkungspflichten der Anbieterinnen determiniert. ${ }^{54}$ Vorliegend ist sie von Interesse, weil sich hieraus bestimmt, ob diese Provider ebenfalls als FDA infrage kommen und damit die Täterqualifikation nach Art. 321 ${ }^{\text {ter }}$ StGB erfüllen.

20 Da OTT-Dienste auf bestehende Netzinfrastrukturen zurückgreifen, ohne Verantwortung für die Informationsübertragung zu übernehmen, erfüllen sie das für die Qualifikation als FDA notwendige Erfordernis des Angebots von Senden oder Empfangen von Informationen an sich nicht. ${ }^{55}$ Bislang fiel die Praxis des Bundesamtes für Kommunikation (BAKOM) indessen dahingehend aus, im Sinne eines funktionalen Verständnisses auch OTTDienste als FDA zu behandeln, sofern diese eine Individualkommunikation ermöglichen. ${ }^{56}$ Auch die Botschaft

50 Urteil des Bundesgerichts 2C_544/2020 vom 29. April 2021 E.4.2.

51 Botschaft zum Bundesgesetz betreffend die Überwachung des Postund Fernmeldeverkehrs (Fn.24), S. 2707.

52 Vgl. LÖTSCHER, Der digitale Nachlass (Fn. 9), S. 80; zum Ganzen auch Urteil des Bundesverwaltungsgerichts A-550/2019 vom 19. Mai 2020 E. 4.; bejahend zunächst BAKOM, siehe EJPD, Dienst Überwachung Post- und Fernmeldeverkehr, Merkblatt «FDA - AAKD», Abgrenzung zwischen Fernmeldedienstanbieterinnen (FDA) und Anbieterinnen abgeleiteter Kommunikationsdienste (AAKD), 10. April 2018, S.3(zit. BAKOM); ablehnend Urteil des Bundesgerichts 2C_544/2020 vom 29. April 2021 E. 3.2.

53 Vgl. Urteil des EuGH C-193/18 vom 13.Juni 2019; siehe auch Urteil des Bundesgerichts 2C_544/2020 vom 29. April 2021 E. 5.1; ebenso Bot schaft zum Bundesgesetz betreffend die Überwachung des Postund Fernmeldeverkehrs (Fn. 24).

54 So kommen FDA etwa umfassende Überwachungspflichten zu (vgl. Art. 21f., Art. 26 BÜPF), während AAKD nur gewisse Duldungs- und Zusammenarbeitspflichten treffen (vgl. Art. 27 BÜPF); vgl. auch Botschaft zum Bundesgesetz betreffend die Überwachung des Postund Fernmeldeverkehrs (Fn. 24), S. 2694.

55 Vgl. Urteil des Bundesgerichts 2C_544/2020 vom 29. April 2021 E.4.2.

56 Siehe BAKOM (Fn. 54), S.3f.; Nachweis auch bei LÖTSCHER, Der digitale Nachlass (Fn.9), S. 80 f. zur Revision des FMG schien noch von einem umfassenden Begriffsverständnis der FDA auszugehen, von welchem auch die OTT-Dienste umfasst seien, wobei jedenfalls die aktiven Auskunft- und Aufsichtspflichten des BÜPF hiervon unberührt bleiben sollten. ${ }^{57}$

Entgegen dieser Auffassung erging jedoch jüngst ein 21 Urteil des Bundesgerichts, in welchem dieses entschied, dass Anbieter von Messaging-Diensten, welche weder direkt noch indirekt einen Internetzugang anbieten und Kunden gegenüber keine Verantwortung für die Informationsübertragung über das Internet übernehmen, nicht unter den Begriff der FDA fallen. ${ }^{58}$ Dieser Entscheid hat Auswirkungen auf die Beurteilung der zu den OTTDiensten zählenden zahlreichen reinen E-Mail-Provider. Es ist davon auszugehen, dass diese nach Auffassung des Bundesgerichts nicht als FDA zu qualifizieren sind, sodass eine Strafbarkeit nach Art. $321^{\text {ter }}$ StGB nach vorliegender Auffassung ausser Betracht fallen würde.

Für den Bereich der unterschiedlichen Mitwirkungs- und 22 Überwachungspflichten erscheint die Differenzierung zwischen FDA und AAKD sowie den hierzu zählenden OTT-Diensten angezeigt. Ob diese Ungleichbehandlung jedoch auch aus strafrechtlicher Sicht und mit Blick auf den Schutzzweck des Fernmeldegeheimnisses sachgerecht erscheint, ist durchaus infrage zu stellen. Angesichts des Wortlauts von Art. $321^{\text {ter }}$ StGB ist sie jedoch im Lichte des strafrechtlichen Legalitätsprinzips de lege lata gesetzlich geboten, da OTT-Dienste aufgrund ihrer Funktionsweise auch nach vorliegender Auffassung nicht als FDA zu qualifizieren sind. ${ }^{59}$ Abhilfe schaffen könnte eine ausdrückliche Aufnahme von AAKD und damit auch der OTT in den Straftatbestand. Wie noch darzulegen sein wird, kommt für die OTT-Dienste ungeachtet dessen jedenfalls eine Strafbarkeit nach Art. $143^{\text {bis }}$ StGB weiter in Betracht.

\section{d) Stellung der Erben als Dritte?}

Selbst wenn man in Anlehnung an die bisherige Praxis des 23 BAKOM von einer funktionalen Gleichstellung von FDA und OTT-Diensten ausgeht, bleibt ferner zu beachten, dass die Handlungen nur tatbestandsmässig i.S.v. Art. $321^{\text {ter }}$ StGB sind, wenn sie gegenüber einem Dritten erfolgen. Ob Erben eine diesbezügliche Drittstellung zukommt, scheint im Schweizer Schrifttum bislang kaum diskutiert. ${ }^{60}$ Auf diese Frage ist daher zurückzukommen. ${ }^{61}$

57 Vgl. Botschaft des Bundesrates vom 6. September 2017 zur Revision des Fernmeldegesetzes (BBl 20176559), S. 6660.

58 Siehe Urteil des Bundesgerichts 2C_544/2020 vom 29. April 2021 E.4.2, E. 5 («Threema»); ebenso bereits Urteil des Bundesverwaltungsgerichts A-550/2019 vom 19. Mai 2020 E. 4, insbes. E. 4.10 (Vorinstanz).

59 Vgl. Urteil des Bundesgerichts 2C_544/2020 vom 29. April 2021 E. $4 \mathrm{f}$. 60 Vgl. LÖTSCHER, Das erbrechtliche Schicksal (Fn.10), S. 319. 61 Siehe nachfolgend III. 


\section{Unbefugtes Eindringen in ein Datenverarbeitungssystem (Art. 143 ${ }^{\text {bis }}$ StGB)}

Gemäss Art.143 bis Abs.1 StGB wird auf Antrag bestraft, wer ohne Bereicherungsabsicht auf dem Wege von Datenübertragungseinrichtungen unbefugterweise in ein fremdes, gegen seinen Zugriff besonders gesichertes Datenverarbeitungssystem eindringt. Tatobjekt ist die Datenverarbeitungsanlage selbst und nicht die darin gespeicherten Daten. ${ }^{62}$ Ein Datenverarbeitungssystem ist dann gegen Zugriff besonders gesichert, wenn der Zugang etwa durch Passwörter geschützt ist. ${ }^{63}$ Passwortgeschützte E-Mail-Accounts einer anderen Person stellen Teile eines fremden Datenverarbeitungssystems und damit ein taugliches Tatobjekt dar. ${ }^{64}$ Der Täter dringt in das Datenverarbeitungssystem ein, wenn er die Zugriffsschranken aktiv ausschaltet oder überwindet. ${ }^{65}$ Unerheblich ist, wie der Täter Zugang zum Passwort erhalten hat. ${ }^{66}$ Vollendet ist die Tat mit der Überwindung der Zugriffsschranke und dem Zugriff auf das System, d.h. hier mit dem unbefugten Einloggen in den fremden E-Mail-Account. ${ }^{67}$

\section{a) Inverkehrbringen von Passwörtern als Tathandlung nach Art. 143 ${ }^{\text {bis }}$ Abs. 2 StGB}

Als Vorbereitungstatbestand ${ }^{68}$ stellt Art. $143^{\text {bis }}$ Abs. 2 StGB unter anderem das Inverkehrbringen oder Zugänglichmachen von Zugangsdaten wie Passwörtern, bei welchen der Täter annehmen muss, dass diese zum unbefugten Eindringen in ein Datenverarbeitungssystem verwendet werden sollen, unter Strafe. Unerheblich ist, ob ein späterer Zugriff tatsächlich erfolgt, da es sich bei Art. $143^{\text {bis }}$ Abs. 2 StGB um ein abstraktes Gefährdungsdelikt handelt. ${ }^{69}$ Anders als bei Abs. 1 handelt es sich zudem um ein Offizialdelikt. ${ }^{70}$ Wie in Art. $143^{\text {bis }}$ Abs. 1 StGB genügt auch hier auf subjektiver Ebene bereits eventualvorsätzliches Handeln zur Begründung der Strafbarkeit. ${ }^{71}$ Die Preisgabe von Passwörtern durch die Provider an eine unbe-

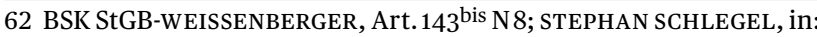
Wohlers/Godenzi/Schlegel (Hrsg.), Schweizerisches Strafgesetzbuch, Handkommentar, Bern 2020, Art. 143 ${ }^{\text {bis }}$ N 2 (zit. HK StGB-BEARBEITERIN).

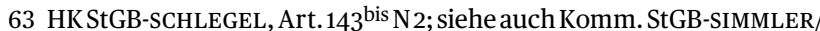
SELMAN, Art.143 ${ }^{\text {bis N } 4 \text {; BGE145IV } 185 \text { E. 2.1. }}$

64 BGE145IV 185 E. 2.2.1.; siehe auch BSK StGB-WEISSENBERGER, Art. $143^{\text {bis }}$ N19; Komm. StGB-SIMMLER/SELMAN, Art. $143^{\text {bis }} \mathrm{N}_{3}$.

65 HKStGB-SCHLEGEL, Art.143 ${ }^{\text {bis }}$ 3 3 ; Komm. StGB-SIMMLER/SELMAN, Art. $143^{\text {bis }} \mathrm{N} 5$.

66 BGE145IV185 E. 2.2.2.; vgl. auch Komm. StGB-SIMMLER/SELMAN, Art. $143^{\text {bis }} \mathrm{N} 5$.

67 Vgl. etwa BGE145IV185E. 2.1.; Urteil des Bundesgerichts 6B_615/2014 vom 2. Dezember 2014 E. 4.3.

68 Vgl. etwa HKStGB-SCHLEGEL, Art. $143^{\text {bis N } 6 \text {; MARK PIETH, Strafrecht }}$ Besonderer Teil, Basel 2018, S. 153.

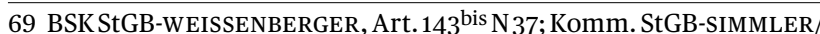
SELMAN, Art. $143^{\text {bis }}$ N11.

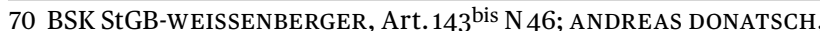
Strafrecht III, 11. Aufl., Zürich 2018, S. 209.

71 Vgl. HKStGB-SCHLEGEL, Art.143 ${ }^{\text {bis N } 8 ; ~ K o m m . ~ S t G B-S I M M L E R / S E L-~}$

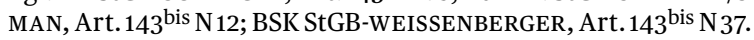

rechtigte Person vermag damit eine taugliche Tathandlung nach Art. $143^{\text {bis }}$ Abs. 2 StGB darzustellen.

\section{b) Unbefugtes Eindringen in ein fremdes Datenverarbeitungssystem - bei Erben einschlägig?}

Eine Strafbarkeit nach Art. $143^{\text {bis }}$ StGB kommt nur in Be- 26 tracht, wenn die infrage stehende Datenverarbeitungsanlage - hier der fragliche E-Mail-Account - für den Empfänger des Passwortes als fremd zu qualifizieren ist und Eindringen unbefugt ist. Massgebend in Bezug auf die Fremdheit ist nach überwiegender Auffassung nicht das sachenrechtliche Eigentumsverhältnis, sondern die fehlende Zugangsberechtigung seitens des Täters. ${ }^{72}$ Unbefugt erfolgt das Eindringen, wenn es gegen den Willen des Verfügungsberechtigten geschieht bzw. der Täter nicht zum Zugriff berechtigt ist. ${ }^{73}$ Dies ist etwa beim Eindringen in ein fremdes passwortgeschütztes E-Mail-Konto durch die Eingabe des Passwortes ohne diesbezügliche Berechtigung der Fall. ${ }^{74}$

Gegenüber Dritten sind diese Voraussetzungen unzwei- 27 felhaft erfüllt, sodass sich Provider bei einer Herausgabe der Zugriffsdaten an Dritte nach Art. $143^{\text {bis }}$ Abs. 2 StGB strafbar machen. Demgegenüber stellt sich in Bezug auf die Erben mit Blick auf deren Sonderstellung die Frage, ob bei diesen ebenfalls von einem unbefugten Eindringen in ein fremdes Datenverarbeitungssystem ausgegangen werden kann oder ob sie als neue Zugriffsbefugte zu betrachten sind. ${ }^{75}$

\section{Vererbbarkeit des digitalen Nachlasses}

Wie die vorangehende Untersuchung gezeigt hat, ist eine 28 Auseinandersetzung mit der Zugriffsberechtigung der Erben auf die E-Mail-Accounts vorliegend von grundlegender Bedeutung. Erst vor diesem Hintergrund lässt sich eine allfällige Strafbarkeit abschliessend beurteilen.

Nach Art. 560 ZGB geht der gesamte Nachlass, d.h. sämt- 29 liche vererbbaren Vermögenswerte, mittels Universalsukzession von Gesetzes wegen an die Erben über. ${ }^{76}$ Nach

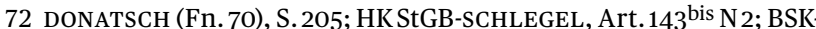
WEISSENBERGER, Art. $143^{\text {bis }}$ N12; im Ergebnis zustimmend auch PK StGB-TRECHSEL/CRAMERI, Art. $143^{\text {bis }}$ N 4 ff., darauf hinweisend, dass dem Adjektiv «fremd» neben «unbefugterweise» aber somit eigentlich keine eigenständige Bedeutung mehr zukomme.

73 Vgl. GÜNTER STRATENWERTH / GUIDO JENNY / FELIX BOMMER (Hrsg.) Schweizerisches Strafrecht, Besonderer Teil I, 7. Aufl., Bern 2010, \$14 N40; HKStGB-SCHLEGEL, Art. $143^{\text {bis N } 2 .}$

74 Vgl. BGE145IV185 E. 2.2.1.

75 Vgl. nachfolgend III.

76 Vgl. hierzu Ivo schWANDER, in: Geiser/Wolf(Hrsg.), Basler Kommentar, Zivilgesetzbuch II, 6. Aufl., Basel 2019, Art. 560 ZGB N1f. 
h.L. gilt dies auch für den digitalen Nachlass, ${ }^{77}$ worunter auch E-Mails sowie die Vertragsbeziehungen zu den Providern fallen. ${ }^{78}$

30

Abgerufene E-Mails, welche ausschliesslich auf einem lokalen Datenträger - etwa dem Computer des Erblassers - gespeichert und vom externen Server gelöscht wurden, gehen gemeinsam mit dem Datenträger an die Erben über. ${ }^{79}$ Eine Unterscheidung zwischen E-Mails mit Inhalten privater und vermögensrechtlicher Natur wird in der Literatur mit dem Verweis auf die physische Briefpost, welche unabhängig von allfälligen privaten Inhalten vererbt wird, ${ }^{80} \mathrm{zu}$ Recht abgelehnt. ${ }^{81}$

Fraglich ist jedoch, wie sich die Rechtslage bei E-Mails gestaltet, die sich auf dem externen Server des Providers befinden. Reine Daten stellen nach vorherrschender Auffassung keine Sachen dar und fallen damit nicht unter Art. 560 ZGB. ${ }^{82}$ Grundsätzlich vererbbar sind demgegenüber die als schuldrechtliche Verhältnisse ausgestalteten Nutzungsvereinbarungen zwischen dem AccountNutzer und dem Provider. ${ }^{83}$ Für die zumeist als Innominatkontrakte ausgestalteten Nutzungsverträge ${ }^{84}$ besteht mangels gesetzlicher Regelung grundsätzlich kein gesetzlicher Ausschluss der Vererbbarkeit. ${ }^{85}$ Folglich wird auch der Nutzungsvertrag des Erblassers mit dem Provider von der Universalsukzession erfasst. ${ }^{86}$ Die Erben treten damit in die Rechtsstellung des Erblassers als AccountInhaber ein. ${ }^{87}$ Dies gilt auch dann, wenn den Erben das Passwort nicht ausdrücklich durch den Erblasser überlassen wurde. ${ }^{88}$ In der Folge gehen sämtliche vertraglichen Rechte und Pflichten an die Erben über, wozu namentlich auch das Recht auf Zugriff auf den Account zählt, zumal dies gerade die vertragliche Hauptleistungspflicht

77 Statt vieler MATTHIAS HÄUPTLI, in: Abt/Weibel (Hrsg.), Praxiskommentar Erbrecht, 4. Aufl., Basel 2019, Art. 560 ZGB N 2 (zit. PK Erbrecht-BEARBEITERIN); BRUCKER-KLEY et al. (Fn. 8), S. 40; KÜNZLE (Fn. 4), S.39f.; BOSSHARDT/BUR (Fn. 2), S.181.

78 Siehe HRUBESCH-MILlaUER (Fn. 9), S.124 ff.; LÖTSCHER, Der digitale Nachlass (Fn. 9), S. 5; KÜNZLE (Fn. 4), S. 39.

79 HÜRLIMANN/HÄNSENBERGER (Fn.11), S.69; HRUBESCH-MILLAUER (Fn. 9), S.129; KÜNZLE (Fn. 4), S. 43.

80 Siehe auch HÜRLIMANN/HäNSENBERGER (Fn.11), S. 69.

81 HRUBESCH-MILLAUER (Fn. 9), S.137; KÜNZLE (Fn. 4), S. 43.

82 Siehe HÜRLIMANN/HÄNSENBERGER (Fn.11), S.71 m.H.; vgl. etwa auch DANIEL HÜRLIMANN / HERBERT ZECH, Rechte an Daten, sui generis 2016, N 8; a.M. offenbar KÜNZLE (Fn. 4), S. 39, nach welchem Daten vom digitalen Nachlass erfasst zu sein scheinen.

83 Siehe LÖTSCHER, Das erbrechtliche Schicksal(Fn. 10), S. 310; HÜRLIMANN/HÄNSENBERGER (Fn.11), S. 82.

84 Vgl. SO HÜRLIMANN/HÄNSENBERGER (Fn.11), S. 72; LÖTSCHER, Da erbrechtliche Schicksal (Fn.10), S.311.

85 LÖTSCHER, Das erbrechtliche Schicksal (Fn.10), S. 311.

86 HRUBESCH-MILLAUER (Fn. 9), S. 136; KÜNZLE (Fn. 4), S. 39.

87 LÖTSCHER, Das erbrechtliche Schicksal(Fn.10, S. 309; BGE133 III 664 E. 2.5.; BGE132 III 677 E. 4.2.4; vgl. auch KÜNZLE (Fn. 4), S. $39 \mathrm{ff}$.

88 Vgl. BOSSHARDT/BUR (Fn. 2), S. 185. darstellt. ${ }^{89}$ Auch Auskunfts- und Informationsansprüche gehen an die Erben über, sofern diesen keine Geheimhaltungspflichten gegenüberstehen. ${ }^{90}$

Zu prüfen bleibt, ob dem Zugriffsrecht der Erben auf das 32 Benutzerkonto trotz des Übergangs des Nutzungsvertrages im Einzelnen Hindernisse entgegenstehen könnten.

\section{Ausschluss durch AGB?}

Zuweilen finden sich in den AGB von Providern Klauseln, 33 welche die Übertragung des Accounts bzw. die Weitergabe von Zugangsdaten an Dritte ausschliessen. ${ }^{91}$ Dies vermag jedoch kein Hindernis für die Vererbbarkeit des Nutzungsvertrages darzustellen. So wird vertreten, von diesen Klauseln sei nur die Übertragung des Accounts durch Schenkung, Verkauf oder andere Formen der Weitergabe unter Lebenden erfasst und nicht der als Universalsukzession stattfindende Erbvorgang.92 Überdies sind Erben auch nicht als Dritte zu qualifizieren, da sie in den Nutzungsvertrag eintreten, weshalb sie von einer derartigen Klausel ohnehin nicht umfasst sind. ${ }^{93}$ Gültig sein kann hingegen die Abrede über die Löschung der Daten auf dem Account des Inhabers nach dessen Ableben, sofern die Klausel einer AGB-Kontrolle standhält. ${ }^{94}$

\section{Unvererbbarkeit höchstpersönlicher Inhalte?}

Die E-Mails eines Erblassers können mitunter einen 34 höchstpersönlichen Inhalt aufweisen, welcher einer Vererbbarkeit möglicherweise entgegenstehen könnte. Nach Schweizer Recht gilt das Prinzip der Unvererbbarkeit höchstpersönlicher Rechte und Pflichten. ${ }^{95}$ Indessen gelten Nutzungsverträge ungeachtet eines allfällig höchstpersönlichen Inhalts insgesamt nicht als Verträge höchstpersönlicher Natur. ${ }^{96}$ Dies liegt mitunter daran, dass regel-

89 Vgl. BOSSHARDT/BUR (Fn. 2), S. 181; HRUBESCH-MILLAUER (Fn. 9), S.131f.

90 Vgl. LÖTSCHER, Das erbrechtliche Schicksal(Fn. 10), S.310 f.; PK Erbrecht-HÄUPTLI, Art. $560 \mathrm{~N} 16$.

91 BOSSHARD/BUR (Fn. 2), S. 178; HÜRLIMANN/HÄNSENBERGER (Fn.11), S.79, jeweils m.H.

92 So HÜRLIMANN/HÄNSENBERGER (Fn.11), S. 79.

93 BOSSHARD/BUR (Fn. 2), S. 178; LÖTSCHER, Der digitale Nachlass (Fn. 9), S. 158.

94 Vgl. HÜRLIMANN/HÄNSENBERGER (Fn.11), S. $82 \mathrm{f}$.

95 Vgl. hierzu statt vieler TARKAN GÖKsU, in: Amstutz et al. (Hrsg.), Handkommentar zum Schweizer Privatrecht, Erbrecht, 3. Aufl., Zürich 2016, Art. 560 ZGB N 9; LÖTSCHER, Der digitale Nachlass (Fn. 9), S. 74; HÜRLIMANN/HÄNSENBERGER (Fn.11), S. 72, S. 82 f.; BGE133 III 664 E. 2.5; Urteil des Bundesgerichts 2C_586/2015 vom 9. Mai 2016 E.3.2. (nicht publ. in BGE142 II307).

96 Siehe LÖTSCHER, Der digitale Nachlass (Fn. 9), S. 75; HRUBESCH-MILLAUER (Fn. 9), S.131; so auch Urteil des Bundesgerichtshofs IIIZR183/17 vom 12. Juli 2018 N 33 ff. (Facebook-Account). Bedeutsam ist der höchstpersönliche Charakter demgegenüber für den Umfang der Nutzungsberechtigung der Erben, siehe hierzu etwa LÖTSCHER, Der digitale Nachlass (Fn. 9), S. 76. 
mässig standardisierte Nutzungsverträge verwendet werden, die gerade nicht persönlich-individuell auf den Inhaber zugeschnitten sind. ${ }^{97}$ Selbst allfällige Persönlichkeitsrechte des Kommunikationspartners ändern nichts am fehlenden höchstpersönlichen Charakter. Indem dieser die E-Mail absendet, muss er vielmehr hinnehmen, nicht mehr darüber bestimmen zu können, wer letztlich von deren Inhalt Kenntnis erlangt. ${ }^{98}$

Im Schrifttum wird zudem darauf hingewiesen, dass physische Dokumente höchstpersönlichen Inhalts wie Tagebücher und Briefe ebenfalls vererbt werden können.99 Bei der Vererbbarkeit in Bezug auf den Inhalt nicht zwischen physischen und elektronischen Briefen zu unterscheiden, erscheint sachgerecht. ${ }^{100}$ Allfällige höchstpersönliche Inhalte in den E-Mails wirken sich somit nicht auf die Vererbbarkeit der Nutzungsverträge aus.

\section{Fortbestand des Fernmeldegeheimnisses gegenüber Kommunikationspartnern?} entfaltet, ${ }^{102}$ besteht dieses gegenüber Dritten auch nach dem Ableben des Erblassers fort. Bei der Beurteilung, ob eine Geheimnisherrschaft an die Erben übergeht, wird grundsätzlich darauf abgestellt, ob es sich um Informationen überwiegend vermögensrechtlicher oder persönlichkeitsbezogener Art handelt. ${ }^{103}$ So wird ein Übergang der Geheimnisherrschaft etwa beim Bank- oder Steuergeheimnis befürwortet, beim Arzt- und Anwaltsgeheimnis demgegenüber abgelehnt. ${ }^{104}$

97 Siehe LÖTSCHER, Der digitale Nachlass (Fn. 9), S. 74 f.; HRUBESCHMILLAUER (Fn.9), S.131.

98 Urteil des Bundesgerichtshofs III ZR 183/17 vom 12.Juli 2018 N39ff.; Nachweis bei LÖTSCHER, Der digitale Nachlass (Fn. 9), S. 77. Unberührt bleibt indes der daten-und persönlichkeitsrechtliche Schutz der Kommunikationspartner vor der Weitergabe von Kommunikationsinhalten durch die Erben an Dritte, vgl. HÜRLIMANN/HÄNSENBERGER (Fn. 11), S. 72; ferner BRUCKER-KLEY et al. (Fn. 8), S. 56.

99 Siehe BosShaRdT/BUR (Fn. 2), S. 187f.; KÜNZLE (Fn. 4), S. 40.; LÖTSCHER, Der digitale Nachlass (Fn. 9), S. 78.

100 Zustimmend HRUBESCH-MILLAUER (Fn. 9), S. 137; hierzu auch LÖTSCHER, Der digitale Nachlass (Fn. 9), S. 75f.

101 PK Erbrecht-HÄUPTLI, Art. 560 N20; Nachweis bei LÖTSCHER, Der digitale Nachlass (Fn. 9), S.79. Zur geplanten Erbrechtsrevision mit Bezug auf den Vorhalt von Berufsgeheimnissen gegenüber Informationsberechtigten (Art. 601a Abs.3 VE-ZGB) weiterführend LÖTSCHER, Der digitale Nachlass (Fn. 9), S. 80.

102 Vgl. vorstehend II.1.b.

103 PK Erbrecht-HÄUPTLI, Art. 560 N 20; so auch LÖTSCHER, Der digitale Nachlass (Fn. 9), S.79.

104 PKErbrecht-HÄUPTLI, Art. 560 N21ff. m.H.; LÖTSCHER, Der digitale Nachlass (Fn. 9), S. 79 m.w.H.
Teilweise wird im Schrifttum dafürgehalten, dass selbst 38 wenn die Erben in die Rechtsstellung des Erblassers eintreten und damit keine Dritten mehr seien, das Fernmeldegeheimnis jedenfalls gegenüber dem Kommunikationspartner des Erblassers fortbestehe. ${ }^{105}$ Dies stehe nicht nur einem Zugriffsrecht seitens der Erben entgegen, sondern führe vielmehr bei der Herausgabe von Zugangsdaten oder Inhalten zur Strafbarkeit der Provider nach Art. $321^{\text {ter }}$ StGB. 106

Diese Auffassung findet im überwiegenden Schrifttum 39 jedoch zu Recht keinen Zuspruch. Stattdessen wird mehrheitlich darauf verwiesen, dass die Erben als Rechtsnachfolger des Erblassers in das Vertragsverhältnis eintreten und damit auch zu den neuen Geheimnisherren werden. ${ }^{107}$ Hiernach sind diese nicht mehr als Dritte zu qualifizieren, sodass eine Strafbarkeit nach Art. 321 ${ }^{\text {ter }}$ StGB ausgeschlossen sei. ${ }^{108}$

Festzuhalten ist dabei, dass sich die Diskussion richtiger- 40 weise einzig auf die sich noch im Kommunikationsvorgang befindlichen E-Mails beziehen kann, zumal nur diese überhaupt vom Fernmeldegeheimnis erfasst sind. Treten die Erben mit dem Erbgang kraft Universalsukzession in den vormaligen Nutzungsvertrag des Erblassers ein, erscheint es ferner nicht sachgerecht, sie als Dritte i.S.v. Art. 43 FMG zu betrachten. ${ }^{109}$ Dies muss nach hier vertretener Auffassung konsequenterweise auch dazu führen, dass diese als neue Kommunikationsbeteiligte der durch das Fernmeldegeheimnis geschützten Kommunikationsvorgänge gelten. ${ }^{110}$

Nicht überzeugend erscheint zwar das teilweise ange- 41 führte Argument, dass die Erben zu den neuen Geheimnisherren würden, weil sie auch Zugriff auf die physische Briefpost oder auf andere abgespeicherte digitale Inhalte erhalten würden. ${ }^{111}$ Diese betreffen nämlich allesamt Inhalte, welchegerade nicht mehr unter das Fernmeldegeheimnis fallen. Der Gesetzgeber hat sich durch das Fernmeldegeheimnis aber gerade bewusst für den Schutz des Kommunikationsvorganges ausgesprochen.

105 So nur bei HÜRLIMANN/HÄNSENBERGER (Fn.11), S. 84 .

106 HÜRLIMANN/HÄNSENBERGER (Fn.11), S. 84; so wohl auch EGGEL/ GUIDOTTI/WOLF (Fn.12), S.115.

107 BOSSHARDT/BUR (Fn. 2), S. 184; diskutiert auch bei HÜRLIMANN/ HÄNSENBERGER (Fn.11), S. 84.

108 So bei BosShaRdT/BUR (Fn. 2), S. 184, S. 200; LÖTSCHER, Der digitale Nachlass (Fn. 9), S. 72, S. 81 m.w.H. auch zum deutschen Recht; vgl. auch HRUBESCH-MILLAUER (Fn. 9), S.136ff.

109 So auch LÖTSCHER, Das erbrechtliche Schicksal (Fn.10), S. 319; BOSSHARD/BUR (Fn. 2), S. 178 m.w.H.

110 Ebenso LÖTSCHER, Der digitale Nachlass (Fn. 9), S. 81f.

111 So aber Urteil des Bundesgerichtshofs IIIZR183/17 vom 12.Juli 2018 N62f.; vgl. auch LÖTSCHER, Der digitale Nachlass (Fn. 9), S. 82. 

in Bezug auf das Fernmeldegeheimnis an die Erben stattdessen vielmehr damit, dass ein Übergang der Geheimnisherrschaft nur im Bereich überwiegend persönlichkeitsbezogener Belange, nicht aber bei vorwiegend vermögensrechtlichen Belangen grundsätzliche Ablehnung findet.112 Aufgrund der vielseitigen Nutzungsfunktionen ist selbst bei einem privaten Gebrauch von E-MailAccounts heutzutage wohl kaum noch von einer rein persönlichkeitsbezogenen Prägung auszugehen. In Erinnerung zu rufen ist die Funktion des E-Mails als Legitimations- und Zugriffsmedium auch in Bezug auf vermögensrechtliche Belange wie etwa E-Banking-Dienste. Für einen auch vermögensrechtlichen Charakter spricht letztlich die Tatsache, dass E-Mails zum digitalen Nachlass gehören, welcher definitionsgemäss das digitale Vermögen umfasst. ${ }^{113}$ Dass vom Erbgang auch die allfällige persönliche Kommunikation erfasst wird, ist hinzunehmen, zumal es nicht den Providern obliegen kann, eine Triage zur Aussonderung von persönlichkeitsbezogenen E-Mails vorzunehmen. ${ }^{114}$ Schliesslich ist auf den Schutzbereich des Fernmeldegeheimnisses hinzuweisen, welcher nicht personenbezogen, sondern sachbezogen die Vertraulichkeit der Kommunikation schützt. Nach der hier vertretenen Ansicht gilt das Fernmeldegeheimnis somit nur gegenüber «echten» Dritten, denen keine Erbenstellung zukommt. Demgegenüber kommt den Erben zumindest ein Zugriffs- und passives Leserecht in Bezug auf die E-Mail-Accounts des Erblassers zu. ${ }^{115}$

\section{Fazit: Folgen für die Strafbarkeit der Provider}

43 Wie die Untersuchung gezeigt hat, erscheinen für die Strafbarkeit der Provider bei der Herausgabe von Passwörtern eines Erblassers grundsätzlich sowohl Art. 321 ${ }^{\text {ter }}$ StGB als auch Art. $143^{\text {bis }}$ Abs. 2 StGB je nach Konstellation zumindest als grundsätzlich einschlägig.

Bezüglich der Strafbarkeit nach Art. 321ter StGB sei zunächst daran erinnert, dass diese gegenüber den E-Mails, die sich nicht mehr im Kommunikationsvorgang befinden, a priori ausser Betracht fällt. ${ }^{116}$ Gleichsam statuiert

112 Vgl. PK Erbrecht-HÄUPTLI, Art. 560 N 21 ff. m.H.; siehe hierzu auch LÖTSCHER, Der digitale Nachlass (Fn. 9), S. 79; BGE133 II 664 E. 2.6. (Bankgeheimnis).

113 Vgl. KÜNZLE (Fn. 4), S. 39; HRUBESCH-MILLAUER (Fn. 9), S.124 m.H.

114 Neben einer gesetzlichen Grundlage mangelt es an der praktischen Umsetzbarkeit einer solchen allfälligen Pflicht, vgl. BossHARDT/BUR (Fn. 2), S.187f.; KÜNZLE (Fn.4), S. 41 f.

115 Vgl. LÖTSCHER, Der digitale Nachlass (Fn.9), S. 87. Ein allfällig darüber hinausgehendes aktives Nutzungsrecht bestimmt sich anhand der Natur des Accounts (höchstpersönlicher Charakter), a.a.O.S.87f.

116 Dies gilt nach hier vertretener Auffassung in Anlehnung an die bundesgerichtliche Rechtsprechung auch für E-Mails auf dem Server
Art. 321 ter StGB nur eine Geheimhaltungspflicht gegenüber Dritten. ${ }^{117}$ Somit stellt Art. $321^{\text {ter }}$ StGB nur die Herausgabe von Zugangsdaten an Dritte unter Strafe, wobei nach geltender Rechtslage vorausgesetzt ist, dass sich der fragliche Provider als FDA qualifizieren lässt. Gegenüber Erben erfährt der E-Mail-Verkehr nach Ableben des Erblassers hingegen keinen strafrechtlichen Schutz durch das Fernmeldegeheimnis. Diese treten mittels Universalsukzession in den vormaligen Nutzungsvertrag des Erblassers und damit nach vorliegender Auffassung auch in die Geheimnisherrschaft ein. ${ }^{118}$ In Bezug auf die sich noch im Kommunikationsvorgang befindenden E-Mails ist somit nach hier vertretener Auffassung ebenfalls davon auszugehen, dass eine Strafbarkeit der Provider nach Art. 321 ter StGB bei der Herausgabe von Passwörtern an die Erben nicht einschlägig ist. Folglich können Provider von den Erben gestellte Zugangsgesuche nicht unter Vorhalt des Fernmeldegeheimnisses abweisen. ${ }^{119}$ Vielmehr sind sie zu einer umfassenden Zugangsgewährung auf den vollständigen Account verpflichtet. Erblasser und deren Kommunikationspartner werden nicht vor einer Kenntnisnahme des Kommunikationsinhaltes durch die Erben geschützt. ${ }^{120}$ Der Erblasser, der dies verhindern will, muss sich zu seinen Lebzeiten um eine Zugriffsregelung bemühen; als sicherste Lösung erscheint, die Löschung des Accounts für den Fall seines Ablebens zu verfügen.

Bei der Herausgabe von Zugangsdaten an Erben ebenfalls 45 ausser Betracht fällt eine Strafbarkeit der Provider nach Art. $143^{\text {bis }}$ Abs. 2 StGB. Mit dem Eintritt in den Nutzungsvertrag des Erblassers und dem Übergang der Zugriffsberechtigung stellen die E-Mail-Accounts wie dargelegt keine Teile eines fremden Datenverarbeitungssystems dar und der Zugriff der Erben auf den Account erfolgt nicht unbefugt und damit nicht tatbestandsmässig i.S.v. Art. 143 ${ }^{\text {bis }}$ Abs.1StGB. ${ }^{121}$ Damit entfällt auch hier eine Strafbarkeit der Provider. Strafbar machen sich Provider demgegenüber nach Art. $143^{\text {bis }}$ Abs. 2 StGB, wenn sie Zugangsdaten an Dritte herausgeben, denen es mangels Erbenstellung an der erforderlichen Zugangsbefugnis

des Providers, die als zugestellt gelten, weil der Erblasser sein E-Mail-Konto vor seinem Ableben abgerufen hat. Doch selbst bei anderweitiger Rechtsauffassung steht dies jedenfalls einer Kenntnisnahme der Erben nicht entgegen.

117 Vgl. auch BOSSHARDT/BUR (Fn. 2), S. 184.

118 Anders HÜRLIMANN/HÄNSENBERGER (Fn.11), S. 84.

119 BOSSHARDT/BUR (Fn. 2), S. 184; WOLF/EGGEL/GUIDOTTI (Fn. 12), S. 115.

120 Vgl. LÖTSCHER, Der digitale Nachlass (Fn.9), S. 81; ebenso Urteil des Bundesgerichtshofs IIIZR 183/17 vom 12.Juli 2018 N 54.

121 BOSSHARDT/BUR (Fn. 2), S. 185; LÖTSCHER, Der digitale Nachlass (Fn. 9), S.169; EGGEL/GUIDOTTI/WOLF (Fn.12), S. 94, verweisend auf die Tatsache, dass sich Erben analog wie bei der geerbten Immobilie nicht des Hausfriedensbruchs auch hier nicht des Bruchs des durch Art. $143{ }^{\text {bis }}$ StGB geschützten «Computerfriedens» strafbar machen. 
fehlt. ${ }^{122}$ Unerheblich ist dabei eine Qualifikation der Provider als FDA.

E-Mail-Provider sind daher gut beraten, sich vorab den Erbenstatus durch Vorweisen etwa einer Erbbescheinigung nach Art. 559 Abs. 1ZGB nachweisen zu lassen, wie dies zuweilen auch bereits der gängigen Praxis einiger Provider bei der Gewährleistung des Zugangs von Erben

122 Siehe ebenso LÖTSCHER, Der digitale Nachlass (Fn. 9), S. 169. auf die E-Mail-Accounts entspricht. ${ }^{123}$ Ferner bleibt abzuwarten, wie die Gerichte künftig die Rolle von den als OTTDiensten zu qualifizierenden reinen E-Mail-Providern im Gefüge der Strafbarkeit nach Art. 321 ter StGB beurteilen werden, um auch für diesen Aspekt die notwendige Rechtssicherheit zu schaffen.

123 Vgl. KÜNZLE (Fn. 4), S. 44; ferner auch LÖTSCHER, Das erbrechtliche Schicksal (Fn.10), S.315. 